\title{
Protective effect of bromfenac sodium on femtosecond laser-assisted cataract surgery via modulating cyclooxygenase- 2 expression
}

\author{
LU LU ${ }^{1,2}$, JIANGYUE ZHAO ${ }^{1,2}$, JING WANG ${ }^{1-3}$, YU QIN ${ }^{1,2}$ and JINGSONG ZHANG ${ }^{1-3}$ \\ ${ }^{1}$ Department of Ophthalmology, The Fourth Affiliated Hospital of China Medical University; \\ ${ }^{2}$ The Key Lenticular Laboratory of Liaoning Province, Shenyang, Liaoning 110059; \\ ${ }^{3}$ Shenyang Aier Excellence Eye Hospital, Shenyang, Liaoning 110000, P.R. China
}

Received February 6, 2020; Accepted May 27, 2020

DOI: $10.3892 / \mathrm{mmr} .2020 .11311$

\begin{abstract}
Cataract is a blinding-caused disease and affects millions of individuals worldwide. Although conventional phacoemulsification (CPCS) has been widely used for treatment of cataract, the incidence of cataract-caused blindness still increased year by year. Recently, femtosecond laser technology has been expanded to variety of clinical applications, including cataract surgery. The present study evaluated the curative effect of bromfenac sodium (BS) after femtosecond laser-assisted cataract surgery (FLACS) and analyzed the mechanism of action. A total of 90 patients were randomly divided into five groups: Group I, conventional phacoemulsification treatment $(\mathrm{CPCS})+$ dexamethasone $(\mathrm{DEX}) /$ tobramycin (TOB); group II, CPCS + bromfenac sodium (BS); group III, Femtosecond laser-assisted cataract surgery (FLACS) + DEX/TOB; group IV, FLACS + BS; and group V, FLACS + pranoprofen. Aqueous humor was collected from these patients post-surgery. For in vitro studies, SRA01/04 cells were irradiated using UV, followed by the collection of culture media and cell lysate. Prostaglandin $\mathrm{E}_{2}\left(\mathrm{PGE}_{2}\right)$ levels, an indicator of inflammation, were measured using ELISA both in vivo and in vitro. In addition, cyclooxygenase (COX) and cleaved caspase-1 p20 expression levels were analyzed using western blotting. The findings suggested that BS was more effective and safer compared with glucocorticoids (GCs) after cataract surgery. BS can protect against post-operative inflammation by inhibiting $\mathrm{PGE}_{2}$ production. Under in vitro conditions BS prevented the SRA01/04 cells from undergoing apoptosis after UV treatment and also suppressed $\mathrm{PGE}_{2}$ release from UV-irradiated SRA01/04 cells by modulating COX-2
\end{abstract}

Correspondence to: Dr Jingsong Zhang, Department of Ophthalmology, The Fourth Affiliated Hospital of China Medical University, 11 Xinhua Street, Shenyang, Liaoning 110059, P.R. China E-mail: cmu4h-zjs@126.com

Key words: bromfenac sodium, cataract surgery, prostaglandin $\mathrm{E}_{2}$, cyclooxygenase-2, apoptosis expression. Furthermore, BS may have an inhibitory effect on the inflammatory form of cell death. Overall, these results indicated that BS could replace existing GCs as a reliable drug for a perioperative period of cataract surgery. It was also identified that the inhibitory effect of $\mathrm{BS}$ on $\mathrm{PGE}_{2}$ production was mediated via the regulation of COX-2.

\section{Introduction}

Cataract is defined as the formation of a dense and cloudy area in the crystalline lens of the eye (1). Visual impairment due to cataract affects $\sim 40,000,000$ of individuals worldwide and has become a major cause of blindness globally (2). Conventional phacoemulsification (CPCS) has been considered as the most prevalent and effective surgical procedure for cataract in past decades (3). However, while this method has been widely used, more than 20 million patients experience blindness due to bilateral cataracts, especially in developing countries (4). In 2001, for the first time, femtosecond laser technology was introduced in clinical practice, and it was used for flap creation in laser in-site keratomileusis (5). Since this introduction, femtosecond laser technology has been expanded to variety of clinical applications, including cataract surgery (6). Femtosecond laser-assisted cataract surgery (FLACS) is more reliable compared with CPCS (7). For instance, more circular and centered capsulorhexis can be created by FLACS to reduce the intraocular lens (IOL) tilt, as well as decentration during IOL implantation (8). FLACS also allows for more effective control of the post-operative astigmatism via the creation of improved quality corneal incisions (9). Moreover, femtosecond laser-assisted pre-fragmentation of the crystalline lens can improve the phacoemulsification power and time consumption during the surgery (10). However, multiple complications such as inflammation and miosis have been reported in several patients with cataract treated by FLACS (11).

Clinically, both glucocorticoids (GCs) and non-steroidal anti-inflammatory drugs (NSAIDs) are used frequently due to their well-known anti-inflammatory effects (12). As these agents have different mechanisms of action, combination 
therapy with GCs and NSAIDS may provide extra benefits; this method has been routinely used for patients undergoing cataract surgery (13). Bromfenac sodium (BS), a potent NSAIDs, has been observed minimal adverse events in number studies, although it was found the risk of corneal compromise when use of BS to preexisting corneal disease (14). The present study evaluated the curative effect of BS after cataract surgery with the replacement of GCs, and the mechanisms of action by treatment of the patients with BS were clarified.

\section{Materials and methods}

Clinical donors. A total of 90 patients (men, 45; women, 45; age, 50-89; mean age, 67.74 99.34 years) from The Fourth Affiliated Hospital of China Medical University (Shenyang, China) between August 2014 and January 2015 were enrolled in the present study. All the experiments were approved by the Ethics Committee of The Fourth Affiliated Hospital of China Medical University (approval no. ChiCTR-TRC-14005114) and informed consent was signed by every study participant.

The patient population did not include corneal diseases, intraoperative complications and other types of eye diseases, such as inflammation, uveitis and glaucoma, or any other pathologies. Patients receiving systemic or topical anti-inflammatory therapy within 1 month before surgery were also excluded. The study participants were randomly divided into five groups: Group I, CPCS $+0.1 \%$ dexamethasone treatment (DEX; Nitto Medic Co., Ltd.)/0.3\% tobramycin (TOB; Nitto Medic Co., Ltd.); group II, CPCS + 0.1\% BS treatment (Senju Pharmaceutical Co., Ltd.); group III, FLACS + $0.1 \%$ DEX/0.3\% TOB; group IV, FLACS + $0.1 \% \mathrm{BS}$; and group V, FLACS + 0.1\% pranoprofen (Senju Pharmaceutical Co., Ltd.). Each of the study groups (I-V) consisted of a total of 22, 22, 19, 24 and 23 patients, respectively. No significant differences in the surgical data of these groups such as age, sex, eye type and nucleus degree of the lens (based on the Emery-Little classification) (15) were observed. Additional clinical information for these patients is presented in Table I.

Surgical treatment. A total of $\sim 150 \mu \mathrm{l}$ of aqueous humor was collected from patients undergoing routine and FLACS. The specimens were collected at the beginning of the surgery and stored at $-80^{\circ} \mathrm{C}$ until used. Cataract surgery was performed by one surgeon and an assistant. Before the surgery, the pupils were dilated using a combination of $0.5 \%$ tropicamide (Mydrin-P; Santen Pharmaceutical Co., Ltd.) with 5\% phenylephrine hydrochloride eyedrops (Neosynesin, Kowa Co., Ltd.), followed by anesthesia and washing using $4 \%$ lidocaine hydrochloride eye drops (Xylocanine; AstraZeneca Co., Ltd.). Patients in groups I and II were treated using a standard CPCS protocol (16). In brief, a $3.0-\mathrm{mm}$-wide clear corneal incision was made, followed by an insertion into the clear cornea to create a self-sealing incision. Subsequently, the foldable acrylic IOL (AcrySof IQ Toric; Alcon, Inc.) was implanted in the capsular bag.

Surgery in patients of the other three groups (III-V) was performed using FLACS with a LenSX Laser system (AcrySof IQ Toric; Alcon, Inc.) and INFINTI Vision System (AcrySof IQ Toric; Alcon, Inc.). In the case of capsulotomy, the diameter was set at $5.0 \mathrm{~mm}$ and the programmed pulse energy was set at $6 \mu \mathrm{J}$ with an incision depth of $300 \mu \mathrm{m}$. The lens was cut into quadrants and the distance of dot/layer and pulse energy were set to $10 / 10 \mu \mathrm{m}$ and $12 \mu \mathrm{J}$, respectively. The corneal primary incision was a 3.0-mm-wide triplanar incision (the distance of dot/layer, $6 / 6 \mu \mathrm{m}$ ) with an anterior side cut angle of $70^{\circ}$ and a posterior side cut angle of $15^{\circ}$. The corneal auxiliary incision was a $1.0-\mathrm{mm}$-wide incision (the distance of dot/layer, $5 / 5 \mu \mathrm{m}$ ) with $6 \mu \mathrm{J}$ of pulse energy. Suction time was recorded by LenSX laser system. A total of $150 \mu 1$ of aqueous flare was collected from the blunt opened clear corneal primary incision under sterile conditions after the laser treatment and immediately stored at $-80^{\circ} \mathrm{C}$ for further analysis. A standard CPCS was used for removing the broken crystalline lenses, followed by implantation of the foldable acrylic IOL (AcrySof IQ Toric; Alcon, Inc.). Then, the anterior chamber was closed by $\sim 2 \mathrm{ml}$ of saline injection.

Perioperative period treatment. Patients in groups II and IV were treated with $0.1 \%$ BS eye drops $\geq 1 \mathrm{~h}$ before the surgery. Post-operatively these subjects were treated with $0.1 \%$ BS eye drops twice daily for 2 months. Patients in these two groups also received $0.5 \%$ levofloxacin eye drops (Santen Pharmaceutical Co., Ltd.) four times per day for 1 month post-operatively to protect from inflammation. Patients from groups I and III were treated with $0.1 \%$ DEX/0.3\% TOB eye drops four times daily for 1 month after surgery followed by replacement of $0.1 \%$ fluorometholone eye drops (Santen Pharmaceutical Co., Ltd.) four times daily for 1 month. Subjects in group V were treated with $0.1 \%$ pranoprofen eye drops (Senju Pharmaceutical Co., Ltd.) four times per day, which was started 3 days before the surgery and continued up to 2 months post-surgery. Moreover, patients in all the study groups received $0.5 \%$ tropicamide phenylephrine eye drops (Santen Pharmaceutical Co., Ltd.) twice daily for 1 week after the surgery.

Clinical outcomes. Corrected distance visual acuity (CDVA) was measured 1 month before and after the surgery (17). In brief, position the patient at a distance of 6 meters from the chart after ensure the illumination on the testing chart. Ask the patient to read from the top to bottom and from the left to right of the chart. Then, record the visual acuity for each eye separately. The conversion of decimal acuity values to $\log$ MAR was used for the calculation of CDVA. Aqueous flare, intraocular pressure (IOP) and corneal thickness measurements were performed postoperatively at 1, 7, 30, 45 and 60 days using FM-600 Laser Flare Meters (KOWA Company, Ltd.), NT-530P Non-Contact Tonometer (Nidek Co. Ltd.) and Pentacam (Oculus $\mathrm{GmbH})$, respectively. Macular thickness was measured using the Spectralis OCT BluePeak module (Heidelberg Engineering, Inc.). The equipment was set as a 3D scan model (Frequency, 40,000 times/sec; Depth, $2.0 \mathrm{~mm}$; Range, $8.8 \times 8.8 \mathrm{~mm}^{2}$ ) and data analysis was automatically performed using HEYEX 2 image management software system (Heidelberg Engineering, Inc.). The severity of the pain was scored from 0 to 4 , where $0=$ no pain/best outcome; $1=$ mild pain/no need for drug intervention/does not affect normal life; $2=$ moderate pain/affects normal life; $3=$ severe pain/unable to live a normal life/appropriate to drug intervention and $4=$ worst outcome/most pain, and was based on the short form of the Brief Pain Inventory (BPI) (18). Pupil 
Table I. Clinical information of the patients.

\begin{tabular}{|c|c|c|c|c|c|c|}
\hline Parameter & $\begin{array}{l}\text { Group I } \\
(n=22)\end{array}$ & $\begin{array}{c}\text { Group II } \\
(n=22)\end{array}$ & $\begin{array}{l}\text { Group III } \\
(n=19)\end{array}$ & $\begin{array}{c}\text { Group IV } \\
(n=24)\end{array}$ & $\begin{array}{c}\text { Group V } \\
(n=23)\end{array}$ & P-value \\
\hline Age (years) & $70.5 \pm 9.70$ & $65.79 \pm 10.47$ & $65.79 \pm 8.21$ & $65.36 \pm 8.29$ & $65.96 \pm 7.19$ & 0.181 \\
\hline Sex (male/female) & $10 / 12$ & $13 / 9$ & $11 / 8$ & $10 / 14$ & $9 / 14$ & 0.577 \\
\hline Eye (left/right) & $8 / 14$ & $7 / 15$ & $8 / 11$ & $12 / 12$ & $11 / 12$ & 0.196 \\
\hline Cataract nuclear grading (Level II) & 0 & 0 & 1 & 0 & 0 & - \\
\hline Cataract nuclear grading (Level III) & 22 & 21 & 17 & 24 & 23 & 0.279 \\
\hline Cataract nuclear grading (Level IV) & 0 & 1 & 1 & 0 & - & - \\
\hline Preoperative CDVA & $0.65 \pm 0.30$ & $0.64 \pm 0.25$ & $0.83 \pm 0.40$ & $0.75 \pm 0.70$ & $0.73 \pm 0.61$ & 0.353 \\
\hline
\end{tabular}

CDVA, corrected visual acuity.

diameter measurement was performed by the same doctor under normal room light illumination. The pupil diameters of patients were measured the using a ruler in the horizontal and vertical directions. The measurement was repeated three times and the average was taken. The pupil reduction ratio after the surgery was calculated as: (Pupil diameter before surgery-pupil diameter after surgery)/pupil diameter before surgery x100. To measure the size of macular thickness, we reconstruct a surface map as a false-color topographic image and divided into 9 map sectors as shown in below. The central subfield macular thickness (CSMT) was defined as the average of the mean thickness within the central $1,000 \mu \mathrm{m}$ ring. The inner macular ring and the outer macular ring were separated into four quadrants with the diameters of 3,000 and $6,000 \mu \mathrm{m}$, respectively. The macular thickness was calculated as the mean standard \pm deviation in these total 9 regions.

Cell culture and UV B treatment. SRA01/04 (cat. no. RCB1591; RIKEN), a human lens epithelium-derived cell line, was cultured in DMEM (FUJIFILM Wako Pure Chemical) supplemented with 20\% heat-inactivated FBS (MP Biomedicals, LLC) and $1 \%$ penicillin-streptomycin (Nacalai Tesque, Inc.) in a $5 \% \mathrm{CO}_{2}$ humidified incubator at $37^{\circ} \mathrm{C}$. Cells were seeded in 96 -well plate at a density of $5 \times 10^{5}$ cells $/ \mathrm{ml} 1$ day before the experiment, which was followed by overnight incubation at $37^{\circ} \mathrm{C}$. Cells were washed twice with pre-warmed PBS and treated with UV irradiation at room temperature for $30 \mathrm{sec}$ using a CL-1000M UV lamp (Thermo Fisher Scientific, Inc.). Most of the resulting wavelengths were in UVB range 250-365 $\mathrm{nm}$. The used UVB energy sources were at 0,20 , 40,60 and $80 \mathrm{~mJ} / \mathrm{cm}^{2}$. SRA01/04 cells were irradiated for $30 \mathrm{sec}$ in the absence or presence of different concentrations $(0-80 \mu \mathrm{g} / \mathrm{ml})$ of BS at $37^{\circ} \mathrm{C}$. PBS was replaced with fresh DMEM post-UV irradiation.

MTT assay. Viability of SRA01/04 cells was determined using a MTT assay based on mitochondrial reduction of MTT to formazan. Cultured medium was replaced with $200 \mu 1$ MTT-containing fresh medium after the treatment. MTT (FUJIFILM Wako Pure Chemical) solution $(7.5 \mathrm{mg} / \mathrm{ml}$ ) was added into each well ( $20 \mu \mathrm{l} /$ well) followed by incubation at $37^{\circ} \mathrm{C}$ for $1.5 \mathrm{~h}$. Then, $150 \mu \mathrm{l}$ culture supernatant was removed from each well and the formazan crystal was lysed by adding
$100 \mu 1 \mathrm{MTT}$ stop solution $(0.4 \% \mathrm{HCl}, 10 \%$ Triton $\mathrm{X}-100$ in Isopropanol). After $12-\mathrm{h}$ incubation at $37^{\circ} \mathrm{C}$, the absorbance was measured at $570 \mathrm{~nm}$, with $655 \mathrm{~nm}$ as reference wavelength, on a microplate reader (Bio-Rad Laboratories, Inc.).

ELISA. The Prostaglandin $\mathrm{E}_{2}\left(\mathrm{PGE}_{2}\right)$ production in the aqueous flare and culture supernatants after UV exposure were measured using a $\mathrm{PGE}_{2}$ Expression ELISA kit (cat. no. 500141, Cayman Chemical Company) as per the manufacturer's instruction. Human interleukin IL-1 $\beta /$ IL-1F2 DuoSet ELISA kit (cat. no. DY20105, R\&D Systems, Inc.) and Cytotoxicity lactate dehydrogenase (LDH) Assay kit (cat. no. CK12, Dojindo Molecular Technologies, Inc.) were used to detect the release of IL-1 $\beta$ and LDH, respectively, according to the manufacturer's instructions.

Reverse transcription-quantitative PCR (RT-qPCR). Total RNA from SRA01/04 cells was isolated using TRIzol ${ }^{\circledR}$ reagent (Invitrogen; Thermo Fisher Scientific, Inc.) followed by the reverse transcription of extracted RNA at $37^{\circ} \mathrm{C}$ for $1 \mathrm{~h}$ and inactivation of the reaction at $95^{\circ} \mathrm{C}$ for 5 min using a miScript II RT kit (QIAGEN GmbH) in a fluorescence thermal cycler (Bio-Rad Laboratories, Inc.). In brief, the qPCR amplification conditions consisted of pre-denaturation for $3 \mathrm{~min}$ at $94^{\circ} \mathrm{C}$, followed by a total of 30 cycles of denaturation for $30 \mathrm{sec}$ at $94^{\circ} \mathrm{C}$, annealing at $58^{\circ} \mathrm{C}$ for $30 \mathrm{sec}$ and extension for $60 \mathrm{sec}$ at $72^{\circ} \mathrm{C}$. The expression of related genes was measured using a SYBR Green PCR reagent kit (Applied Biosystems; Thermo Fisher Scientific, Inc.) with the following primer sets on an ABIViiA7 RT PCR system (Thermo Fisher Scientific, Inc., Waltham, MA, USA). The relative expression of target genes was normalized to $\beta$-actin. Expression of $\beta$-actin was used as internal control for the analysis of other genes while the $2^{-\Delta \Delta \mathrm{Cq}}$ method was used for data analysis (19). The primers sequences were as following: COX-1 forward, 5'-CTTTTCACCGTA GGTGGCCT-3' and reverse, 5'-AGTGGAAGTGGGCTA CAACG-3'; COX-2 forward, 5'-ACCGTCTGAACTATCCTG CC-3' and reverse, 5'-AGATTAGTCCGCCGTAGTCG-3'; and $\beta$-actin forward, 5'-GTGGGGCGCCCCAGGCACCA-3' and reverse, 5'-CTCCTTAATGTCACGCACGATTTC-3'.

Western blotting. SRA01/04 cells were homogenized in RIPA lysis and extraction buffer (Thermo Fisher Scientific, Inc.). 
A
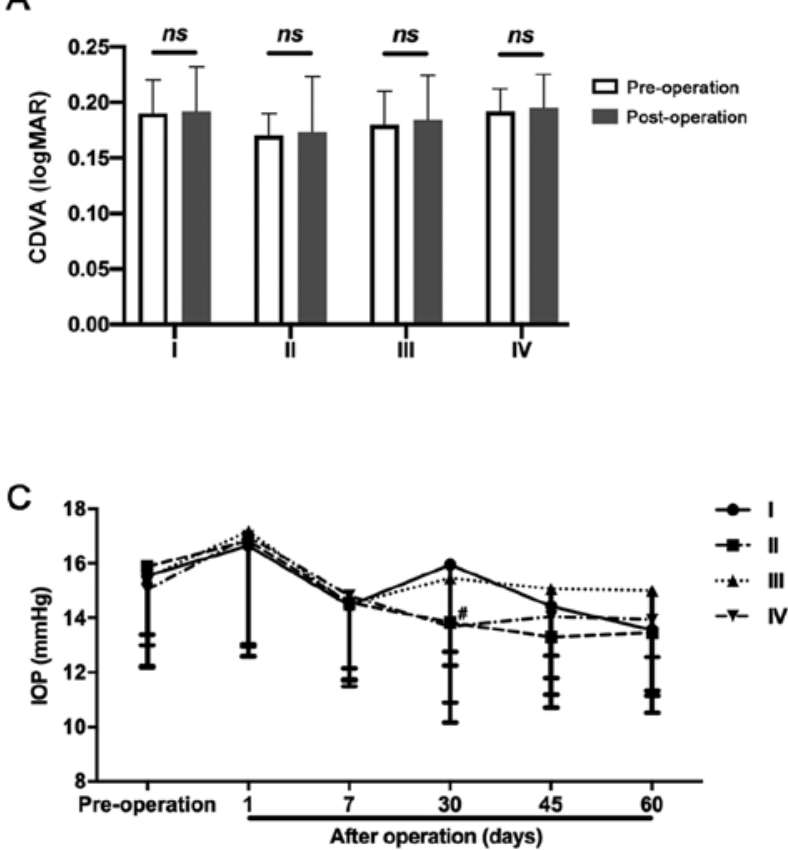

B
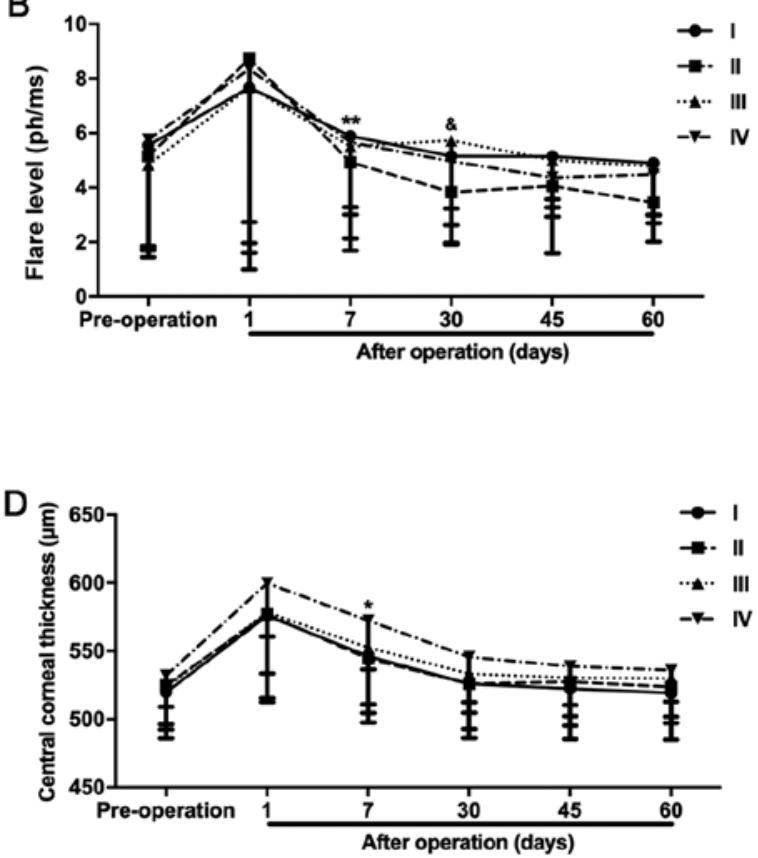

Figure 1. Effect of bromfenac sodium on the perioperative period of cataract surgery. (A) logMAR CDVA values among the groups pre- and 1 week post-operation. Clinical outcomes including (B) aqueous flare, (C) IOP and (D) CCT were measured under both pre and post-operative conditions. data are presented as the mean $\pm \mathrm{SD}$. ${ }^{*} \mathrm{P}<0.05$ group IV vs. group II; ${ }^{\#} \mathrm{P}<0.05$ group II vs. group I; ${ }^{\star} \mathrm{P}<0.05$ group III vs. group II; ${ }^{* *} \mathrm{P}<0.05$ group IV vs. group II. CDVA, Corrected distance visual acuity; IOP, intraocular pressure; CCT, central corneal thickness; ns, not significant.

Bicinchoninic acid Protein assay kit (FUJIFILM Wako Pure Chemical) was used for determining protein concentrations. In total, $20 \mu \mathrm{g}$ each sample were separated by $15 \%$ SDS-PAGE and transferred to PVDF membrane (Immobilon-P; EMD Millipore). Then, 5\% skim milk was used to blocked membranes for $1 \mathrm{~h}$ at room temperature, and membranes were incubated with primary antibodies against cleaved human caspase-1 p20 (cat. no. AG-208-0042-C100; AdipoGen), COX-1 (cat. no. 4841; Cell Signaling Technology, Inc.) and COX-2 (cat. no. 12282; Cell Signaling Technology, Inc.; all 1:1,000 dilutions) overnight at $4^{\circ} \mathrm{C}$. GAPDH (cat. no. 5174; Cell Signaling Technology,Inc.; 1:1,000) was used as an internal control. The secondary horseradish peroxide-conjugated anti-rabbit IgG antibody (Cell Signaling Technology, Inc.; cat. no. 5127; $1: 5,000$ ) was incubated at room temperature for $1 \mathrm{~h}$. Protein band intensity was analyzed using Luminata Forte Western horseradish peroxidase Substrate (EMD Millipore) with a Bio-Rad ChemiDox $\mathrm{XRS}^{+}$imaging system and Image Lab Software Version 6.0.1 (Bio-Rad Laboratories, Inc.).

Statistical analysis. All experiments were conducted with at least three independent replicates. Data are presented as the mean \pm standard deviation using GraphPad Prism 5.0 (GraphPad Software, Inc.). The comparisons of paired samples (preoperative vs. postoperative measurements of the same patient) in Figs. 1 and 2 were assessed using a repeated measures ANOVA, which was used to analyse matched samples. Differences among multiple groups were assessed with one-way ANOVA followed by Bonferroni post hoc test or Scheffe's multiple comparison test (SPSS 22.0; IBM Corp.), which was used to analyse unpaired samples. The relations between categorical variables in Tables I and II were analyzed with the $\chi^{2}$ test. In addition, Fisher's exact test was used to detect differences in levels of cataract between the groups. $\mathrm{P}<0.05$ was considered to indicate a statistically significant difference.

\section{Results}

Beneficial effects of BS on the perioperative period of cataract surgery. No statistically significant differences in the clinical outcomes including CDVA, aqueous flare, IOP, central corneal thickness (CCT) and macular morphology (Table I) among the patients were identified before the surgery. Based on the data in Table II, it was found that the pupil diameter was increased by BS treatment (group II) before the CPCS, as compared with NSAID treatment (group I). However, the pupil diameter was significantly reduced by DEX/TOB treatment when the patients were receiving FLACS (group III). More important, BS treatment strongly improved pupil reduction, as group IV indicated. It was also observed similar protective effect of pranoprofen on pupil reduction as BS. Additionally, cumulative dissipated energy (CDE) in the patients who received FLACS was significantly reduced compared with the patients who received CPCS (Table II), indicating that energy delivered induced less damage in the FLACS group compared with the CPCS group. In addition, no surgical complications in any of the study subjects after the surgery were demonstrated.

Compared with the results of the patients visual acuity before surgery, no significant difference was observed in the visual acuity in patients of each group 1-week post-surgery, suggesting the surgical procedure was successful (Fig. 1A). However, aqueous flare was observed in all the groups 1 day after the surgery (Fig. 1B). Moreover, reduction of the 

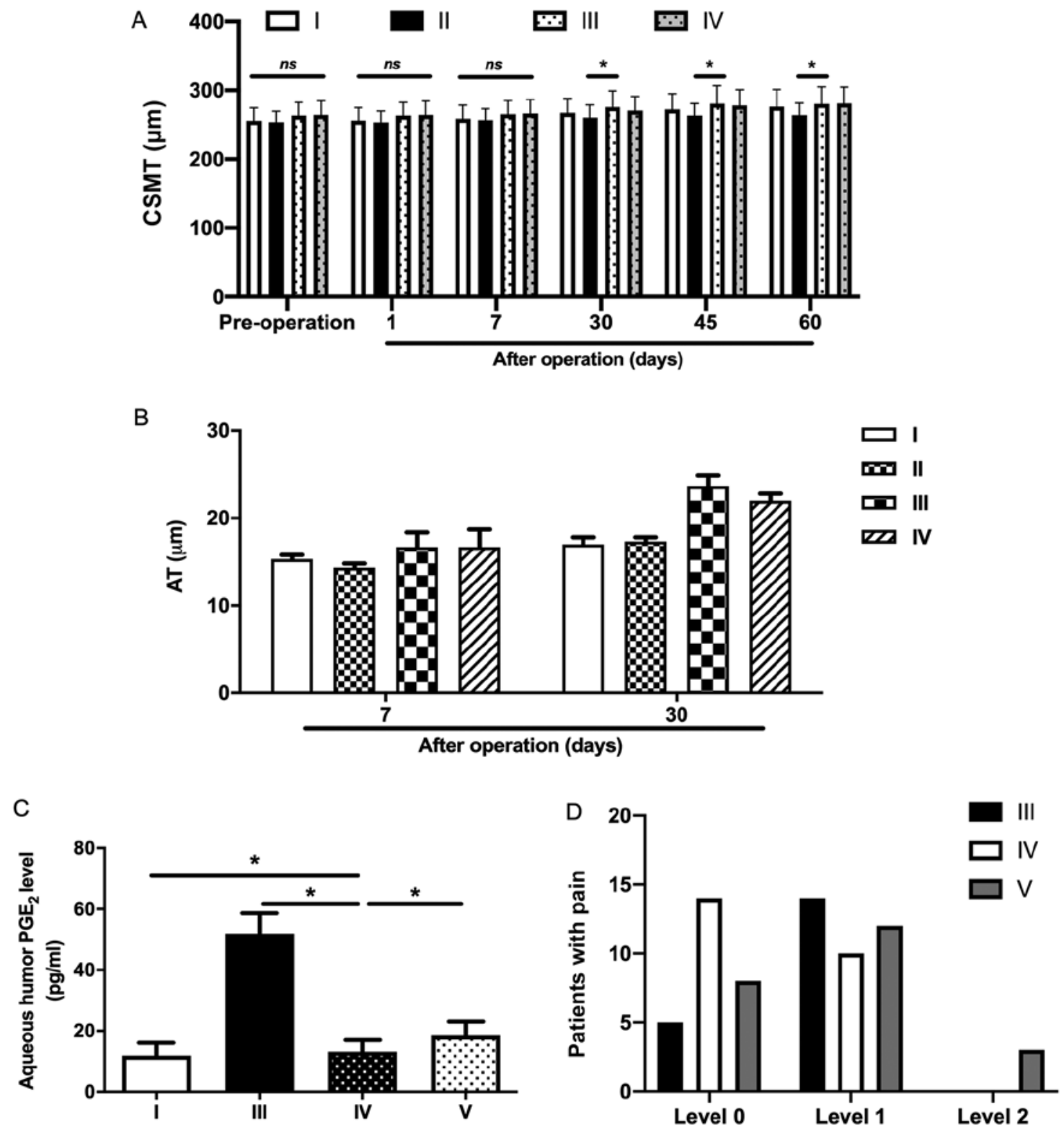

Figure 2. Effects of bromfenac sodium on cataract surgical complications. (A) CSMT changes and (B) inner macular ring AT were measured pre- and post-surgery. (C) $\mathrm{PGE}_{2}$ level in the aqueous humor was quantified by ELISA. (D) The number of patients with pain. Data are presented as the mean \pm SD. ${ }^{*} \mathrm{P}<0.01$. AT, average thickness; CSMT, central subfield macular thickness; $\mathrm{PGE}_{2}$, Prostaglandin $\mathrm{E}_{2}$.

aqueous flare was observed in the BS-treated groups but not in the NSAID-treated groups after 7 days of treatment. It was also found that the aqueous flare was significantly inhibited by BS compared with NSAIDs after 30 days of treatment. Furthermore, significantly higher IOP was observed in GC-treated groups 30 days after surgery as compared with BS-treated subjects (Fig. 1C). It was demonstrated that the CCT value gradually returned to the pre-operative state, although FLACS treatment significantly increased CCT a day after the operation (Fig. 1D).

Protective effects of BS on surgical complications. There were no significant changes of CSMT between each group at 1-week after surgery (Fig. 2A). However, it was observed that BS exerted a protective effect on the expansion of CSMT on days 30, 45 and 60 post-surgery (Fig. 2A). Moreover, on day 30 , the average thickness size of the outer macular ring in FLACS was inhibited by BS (Fig. 2B). It was also found that the inner macular ring AT demonstrated a similar trend to CSMT (data not shown). Thus, BS also reduced the inner macular ring AT in CPCS and FLACS groups.

It has been reported that FLACS can induce the hyperproduction of $\mathrm{PGE}_{2}$, which is one of the major causes of the miosis (20). Therefore, $\mathrm{PGE}_{2}$ levels were measured in each group after the surgery. A higher concentration of $\mathrm{PGE}_{2}$ was detected in the aqueous humor of DEX/TOB-treated FLACS group (group III) compared with that of CPCS group (group I). Additionally, compared with the DEX/TOB-treated patients (group III), either BS treatment (group IV) or pranoprofen treatment (group V) significantly suppressed $\mathrm{PGE}_{2}$ production after the patients received FLACS (Fig. 2C). After FLACS surgery, no severe pain (more than level 2) was found in the patients. As Fig. 2D illustrates, most of patients treated by BS did not felt any pain. However, ten patients received DEX/TOB 


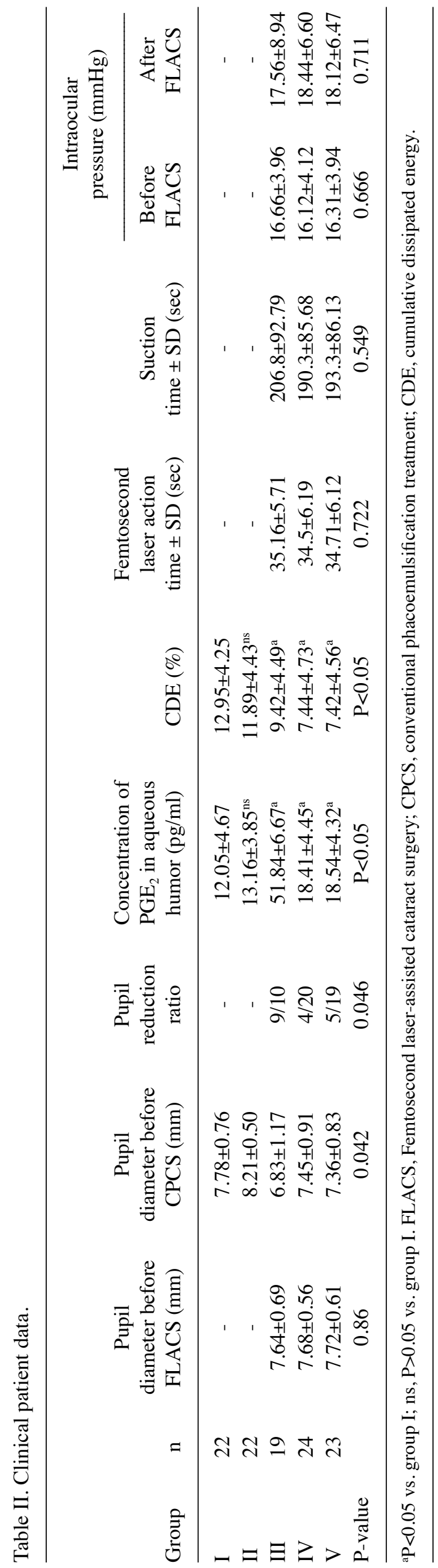

A
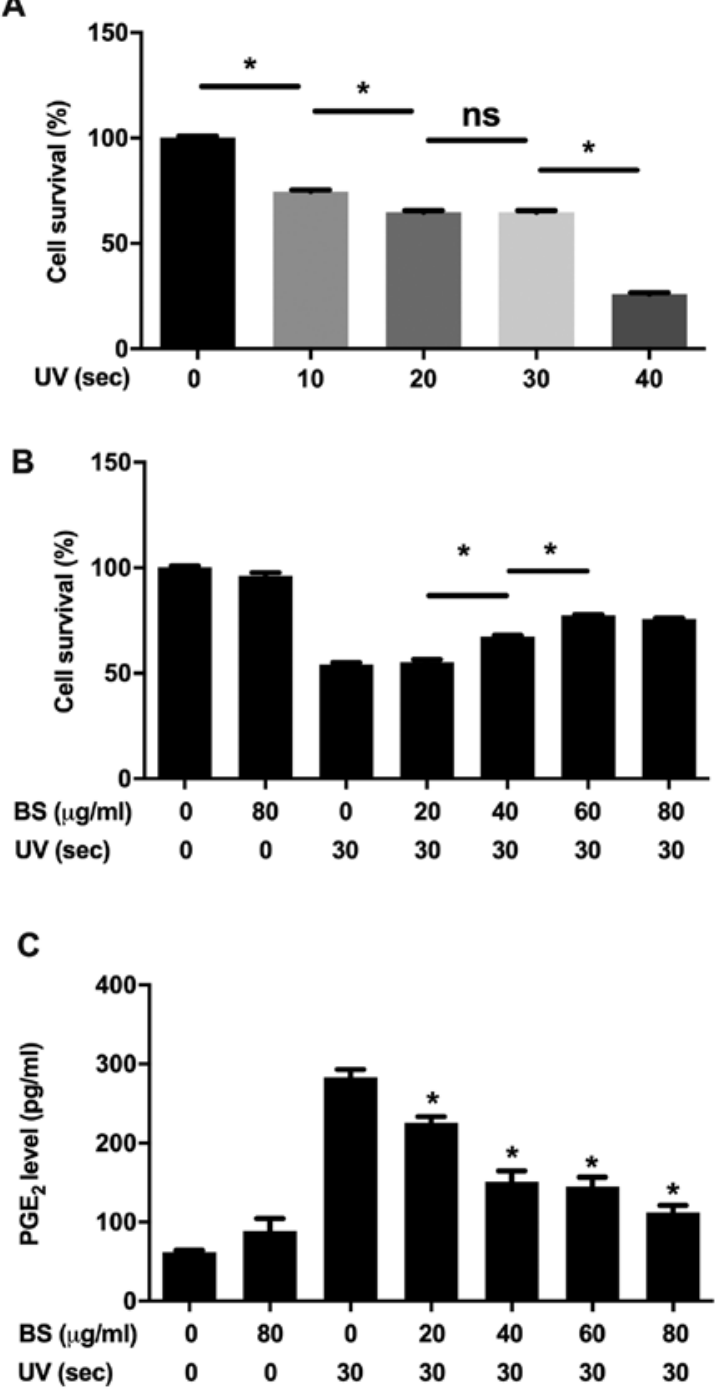

Figure 3. Protective effect of BS on UV-irradiated SRA01/04 cells. (A) To optimize the experimental condition, SRA01/04 cells were exposed to UV $\left(60 \mathrm{~mJ} / \mathrm{cm}^{2}\right)$ as indicated, followed by detection of the cell viability using MTT assay. (B) Protective effect of BS on UV-induced SRA01/04 cells apoptosis was analyzed by MTT assay. (C) Concentrations of $\mathrm{PGE}_{2}$ in the supernatant were measured using ELISA $(C)$. Data are presented as the mean $\pm \operatorname{SD}(n=3)$. ${ }^{*} \mathrm{P}<0.01$. ns, not significant; $\mathrm{PGE}_{2}$, Prostaglandin $\mathrm{E}_{2} ; \mathrm{BS}$, bromfenac sodium.

and 5 patients treated by BS felt mild pain after FLACS surgery. As compared with these two groups, more severe pain (level 2) was found in 3 patients who received pranoprofen treatment. Furthermore, there were 8 patients distributed in level 0 of pain and 12 patients presented in level 1 of pain in pranoprofen group, respectively. Collectively, these results demonstrated that BS has a higher protective effect on pain compared with pranoprofen during the surgery (Fig. 2D).

Protective effects of $B S$ on the perioperative period of cataract surgery via modulating $C O X$. To identify the mechanism underlying the protective effects exerted by BS on the perioperative period of cataract surgery, the present study established an in vitro model via the irradiation of SRA01/04 cells with UV. As demonstrated in Fig. 3A, the apoptosis of SRA01/04 cells occurred in a time-dependent manner post UV irradiation. Additionally, it was identified that $\sim 50 \%$ of cells died after 
A

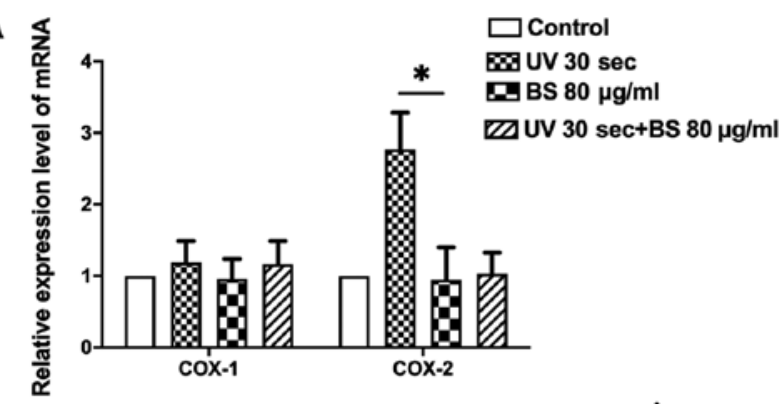

B
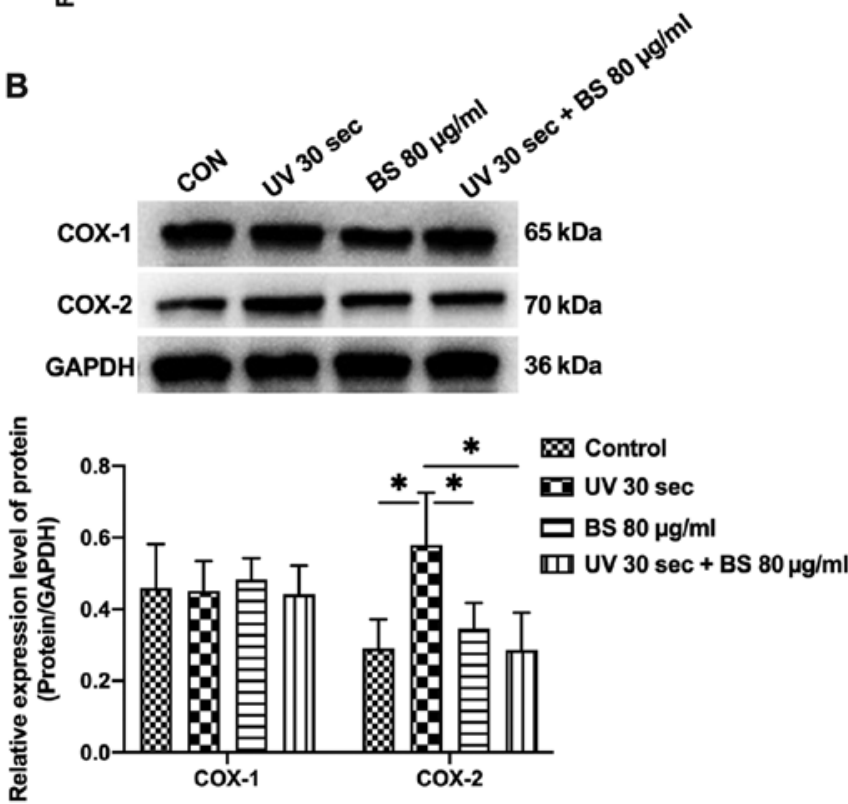

Figure 4. Effect of BS on COX expression in UV-irradiated SRA01/04 cells. (A) In UV-irradiated SRA01/04 cells, the mRNA levels including COX-1 and COX-2 were quantified by reverse transcription-quantitative PCR. (B) Protein expression of COX-1 and COX-2 was analyzed using western blotting. Data are presented as the mean $\pm \mathrm{SD}(\mathrm{n}=3)$. ${ }^{*} \mathrm{P}<0.01$. COX, cyclooxygenase; CON, control; BS, bromfenac sodium.

exposure to UV for $30 \mathrm{sec}$, and that most of the cells died after exposure to UV for $40 \mathrm{sec}$ (Fig. 3A). Moreover, the results suggested that BS prevented SRA01/04 cells from apoptosis in a dose-dependent manner when the cells irradiated with UV for $30 \mathrm{sec}$ (Fig. 3B). Similarly, it was also demonstrated that the $\mathrm{PGE}_{2}$ level in the supernatant was increased by UV exposure compared with non-UV irradiated cells, and this was significantly reversed by BS treatment in a dose-dependent manner (Fig. 3C).

The mRNA expression level of COX-2, but not COX-1, was significantly upregulated by UV irradiation (Fig. 4A). In addition, the protein expression level of COX-2 was significantly increased by UV irradiation (Fig. 4B). The results also indicated that BS treatment significantly inhibited the expression of COX-2 at both transcription and protein level (Fig. 4). Under same condition, the pyroptosis markers including IL-1 $\beta$, LDH and cleaved caspase-1 were enhanced by UV-irradiated cells (Fig. 5). However, treatment of cells with BS strongly suppressed pyroptosis, as accessed by the production of IL-1 $\beta$ and LDH, expression of cleaved caspase-1 as well. These results indicate that BS treatment protects the cell survival via the suppression of pro-inflammatory factors and inhibition of caspase-1 cleavage.

\section{Discussion}

CPCS has been routinely used for the treatment of patients with cataract in the last decade (21). Tissue injuries such as endothelial cell loss and macular edema are frequently induced during surgery (22). Previous studies have reported that the use of FLACS has fewer complications and is more reliable compared with CPCS (23). The present results suggested that there was no difference in CDVA, as well as aqueous flare, between CPCS groups and FLACS groups. Moreover, there was no significant difference in IOP between these groups before or after the surgery, suggesting that the IOP rise after surgery was not associated with several docking attempts, vacuum time and treatment time; these results were consistent with Kerr et al (24). In GC-treated groups, it was found that the IOPs of two patients were not within the normal range (12-22 $\mathrm{mmHg}) 30$ days post-surgery. Furthermore, both aqueous flare and IOP were significantly reduced by BS treatment 30 days after the surgery. Therefore, the present results indicated that CCT was increased by FLACS with BS treatment, which can be interpreted by the frequent use of BS before the surgery.

Macular edema appears usually postoperatively after 1-6 weeks, with a peak in the 4-6th week, and this is considered a major factor for vision impairment after cataract surgery (25). Wittpenn et al (26) have reported that NSAIDs can protect against the occurrence of macular edema and maintain the CCT. The present findings further support this observation and suggest that BS treatment can effectively prevent macular edema in the eyes after cataract surgery. The present results also provided supporting evidence for the effectiveness and safety of BS. Thus, BS may be used as an alternative to GC and it has protective effects on the complications of cataract surgery.

PGEs, a type of lipid autacoids derived from arachidonic acid, have been implicated in a variety of inflammatory diseases such as rheumatoid arthritis or allergic asthma (27). $\mathrm{PGE}_{2}$ is one of the most abundant PGEs in mammals, and is synthesized by COX-1 and -2 (27). Previous studies have reported that overproduction of $\mathrm{PEG}_{2}$ is observed in the aqueous humor of patients after cataract surgery and can cause a reduction in the size of the pupils $(20,28,29)$. $\mathrm{PGE}_{2}$ concentration is significantly reduced in NSAIDs-treated patients as these drugs can directly bind and inhibit the active site of COXs (30). The results of the present study demonstrated that BS, as an NSAID drug, strongly suppresses $\mathrm{PGE}_{2}$ production in patients and in UV-induced cells. However, it should be noted that COX-2 expression, but not COX-1, was inhibited by BS treatment, suggesting that BS mitigates miosis via modulating COX-2.

In addition to $\mathrm{PEG}_{2}$-related inflammation, it has been shown that pyroptosis can play a role in the formation of cataract (31). Pyroptosis, a type of inflammatory cell death, is induced by inflammasome activation, which causes rapid rupture of the cell membrane and the release of pro-inflammatory factors, such as IL-1 $\beta$, IL-18 and LDH (32). In line with these observations, the results of the present study demonstrated that SRA01/04 cells apoptosis was caused by UV irradiation, indicating the presence of UV-induced pyroptosis. The anti-pyroptotoc effects on UV-irradiated cells were observed 
A

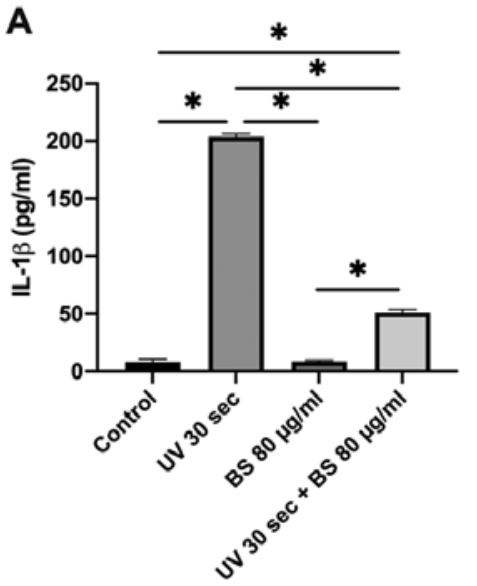

C

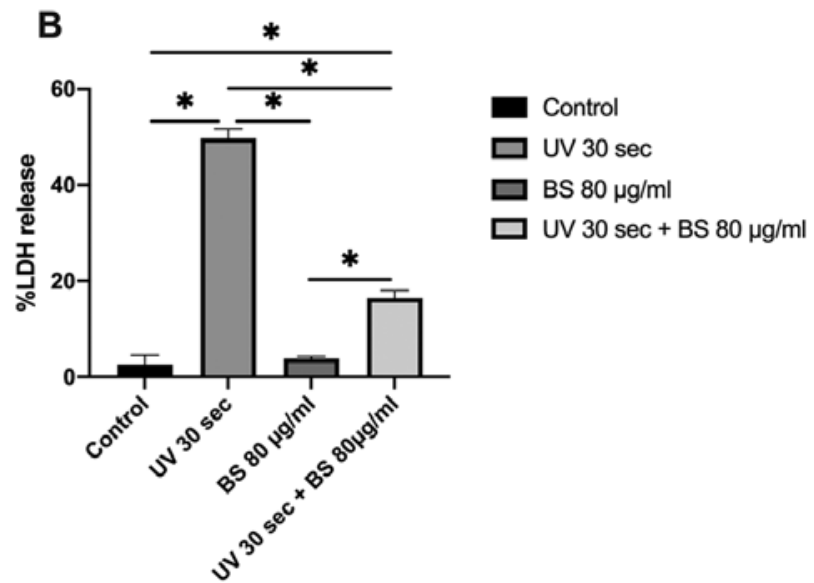

Control

$\square$ UV $30 \mathrm{sec}$

$\square$ BS $80 \mu \mathrm{g} / \mathrm{ml}$

$\square$ UV $30 \mathrm{sec}+\mathrm{BS} 80 \mu \mathrm{g} / \mathrm{ml}$
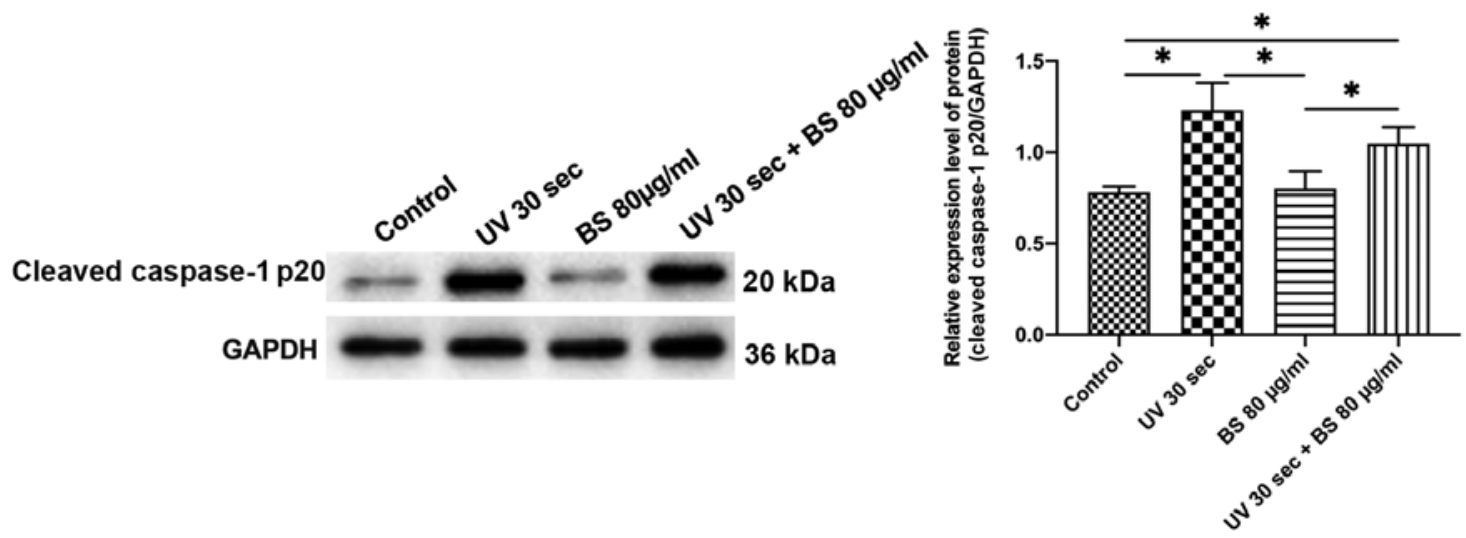

W Control

UV $30 \mathrm{sec}$

E BS $80 \mu \mathrm{g} / \mathrm{ml}$

سل UV $30 \mathrm{sec}+\mathrm{BS} 80 \mu \mathrm{g} / \mathrm{ml}$

Figure 5. Effect of BS on UV-induced SRA01/04 cells pyroptosis. Release of (A) IL-1 $\beta$ and (B) LDH from UV-irradiated SRA01/04 cells was measured by ELISA. (C) Cleaved caspase-1 p20 was analyzed using western blotting. Data are presented as the mean \pm SD $(n=3)$. " $P<0.01$. IL, interleukin; BS, bromfenac sodium; CON, control.

when treatment of cells with BS. To the best of our knowledge, the present study was the first report the inhibitory effects of BS on pyroptosis.

In conclusion, the present study evaluated the curative effect of BS during the perioperative period of cataract surgery and examined its action mechanisms. It was demonstrated that BS was more effective and safer compared with GC after cataract surgery. Furthermore, BS can protect against postoperative inflammation by inhibiting $\mathrm{PGE}_{2}$ production. In vitro BS prevented SRA01/04 cells apoptosis after UV treatment and also suppressed $\mathrm{PGE}_{2}$ release from UV-irradiated SRA01/04 cells by modulating COS-2 expression. Collectively, the present results suggested that BS could replace the existing $\mathrm{GC}$ as a reliable drug for perioperative period of cataract surgery.

\section{Acknowledgements}

Not applicable.

\section{Funding}

This study was supported by the National Natural Science Foundation of China (grant no. 81470617).

\section{Availability of data and materials}

The datasets used and/or analyzed during the current study are available from the corresponding author on reasonable request.

\section{Authors' contributions}

JingsongZ designed this study. LL, JiangyueZ, JW, YQ and Jingsong $Z$ conducted the experiments. LL, JingsongZ and JW analyzed the data. LL and JingsongZ wrote the manuscript. JingsongZ edited the manuscript. All authors read and approved the final manuscript.

\section{Ethics approval and consent to participate}

All the experiments were approved by the Ethics Committee of the Fourth Affiliated Hospital of China Medical University (approval no. ChiCTR-TRC-14005114) and written informed consent was obtained from the participants.

\section{Patient consent for publication}

Not applicable. 


\section{Competing interests}

The authors declare that they have no competing interests.

\section{References}

1. Thompson J and Lakhani N: Cataracts. Prim Care 42: 409-423, 2015.

2. Khairallah M, Kahloun R, Bourne R, Limburg H, Flaxman SR, Jonas JB, Keeffe J, Leasher J, Naidoo K, Pesudovs K, et al: Number of people blind or visually impaired by cataract worldwide and in world regions, 1990 to 2010. Invest Ophthalmol Vis Sci 56: 6762-6769, 2015.

3. Dick HB and Schultz T: A review of laser-assisted versus traditional phacoemulsification cataract surgery. Ophthalmol Ther 6 : 7-18, 2017.

4. Pascolini D and Mariotti SP: Global estimates of visual impairment: 2010. Br J Ophthalmol 96: 614-618, 2012.

5. Nordan LT, Slade SG, Baker RN, Suarez C, Juhasz T and Kurtz R: Femtosecond laser flap creation for laser in situ keratomileusis: Six-month follow-up of initial U.S. clinical series. J Refract Surg 19: 8-14, 2003.

6. Farid M and Steinert RF: Femtosecond laser-assisted corneal surgery. Curr Opin Ophthalmol 21: 288-292, 2010.

7. Whang WJ, Yoo YS, Joo CK and Yoon G: Comparison of refractive outcomes between femtosecond laser-assisted cataract surgery and conventional cataract surgery. Medicine (Baltimore) 97: e13784, 2018.

8. Kránitz K, Miháltz K, Sándor GL, Takacs A, Knorz MC and Nagy ZZ: Intraocular lens tilt and decentration measured by Scheimpflug camera following manual or femtosecond laser-created continuous circular capsulotomy. J Refract Surg 28: 259-263, 2012

9. Pereira A, Somani S, Tam ES, Chiu H and Maini R: Comparison of surgically induced astigmatism and corneal morphological features between femtosecond laser and manual clear corneal incisions. J Refract Surg 35: 796-802, 2019.

10. Nagy Z, Takacs A, Filkorn T and Sarayba M: Initial clinical evaluation of an intraocular femtosecond laser in cataract surgery. J Refract Surg 25: 1053-1060, 2009.

11. Nagy ZZ, Takacs AI, Filkorn T, Kránitz K, Gyenes A, Juhász É, Sándor GL, Kovacs I, Juhász T and Slade S: Complications of femtosecond laser-assisted cataract surgery. J Cataract Refract Surg 40: 20-28, 2014

12. Juthani VV, Clearfield E and Chuck RS: Non-steroidal anti-inflammatory drugs versus corticosteroids for controlling inflammation after uncomplicated cataract surgery. Cochrane Database Syst Rev 7: CD010516, 2017.

13. Hirneiss C, Neubauer AS, Kampik A and Schönfeld CL: Comparison of prednisolone $1 \%$, rimexolone $1 \%$ and ketorolac tromethamine $0.5 \%$ after cataract extraction: A prospective, randomized, double-masked study. Graefes Arch Clin Exp Ophthalmol 243: 768-773, 2005.

14. Jones $\mathbf{J}$ and Francis P: Ophthalmic utility of topical bromfenac, a twice-daily nonsteroidal anti-inflammatory agent. Expert Opin Pharmacother 10: 2379-2385, 2009.

15. Emery JM and Little JH: Patient selection. In: Phacoemulsification and Aspiration of Cataract. C. V. Mosby, St. Louis, MO, pp45-48, 1979.
16. Kelman CD: Phaco-Emulsification and Aspiration: A New Technique of Cataract Removal: A Preliminary Report. Am J Ophthalmol 191: 2018.

17. Marsden J, Stevens S and Ebri A: How to measure distance visual acuity. Community Eye Health 27: 16, 2014

18. Daut RL, Cleeland CS and Flanery RC: Development of the Wisconsin Brief Pain Questionnaire to assess pain in cancer and other diseases. Pain 17: 197-210, 1983.

19. Livak KJ and Schmittgen TD: Analysis of relative gene expression data using real-time quantitative PCR and the 2(-Delta Delta C(T)) method. Methods 25: 402-408, 2001.

20. Schultz T, Joachim SC, Kuehn M and Dick HB: Changes in prostaglandin levels in patients undergoing femtosecond laser-assisted cataract surgery. J Refract Surg 29: 742-747, 2013.

21. Parihar J, Sahoo PK, Dash RG and Kamath AP: An advanced cataract surgery by phacoemulsification: An initial experience. Med J Armed Forces India 54: 229-231, 1998.

22. Dick HB, Kohnen T, Jacobi FK and Jacobi KW: Long-term endothelial cell loss following phacoemulsification through a temporal clear corneal incision. J Cataract Refract Surg 22: 63-71, 1996.

23. Zhang X, Yu Y, Zhang G, Zhou Y, Zhao G, Chen M, Wang Y, Zhu S, Zhang $\mathrm{H}$ and Yao K: Performance of femtosecond laser-assisted cataract surgery in Chinese patients with cataract: A prospective, multicenter, registry study. BMC Ophthalmol 19: 77, 2019.

24. Kerr NM, Abell RG, Vote BJ and Toh T: Intraocular pressure during femtosecond laser pretreatment of cataract. J Cataract Refract Surg 39: 339-342, 2013.

25. Flach AJ: The incidence, pathogenesis and treatment of cystoid macular edema following cataract surgery. Trans Am Ophthalmol Soc 96: 557-634, 1998.

26. Wittpenn JR, Silverstein S, Heier J, Kenyon KR, Hunkeler JD and Earl M; Acular LS for Cystoid Macular Edema (ACME) Study Group: A randomized, masked comparison of topical ketorolac $0.4 \%$ plus steroid vs steroid alone in low-risk cataract surgery patients. Am J Ophthalmol 146: 554-560, 2008.

27. Ricciotti E and FitzGerald GA: Prostaglandins and inflammation. Arterioscler Thromb Vasc Biol 31: 986-1000, 2011.

28. Solomon KD, Turkalj JW, Whiteside SB, Stewart JA and Apple DJ: Topical $0.5 \%$ ketorolac vs $0.03 \%$ flurbiprofen for inhibition of miosis during cataract surgery. Arch Ophthalmol 115: 1119-1122, 1997.

29. Gimbel HV: The effect of treatment with topical nonsteroidal anti-inflammatory drugs with and without intraoperative epinephrine on the maintenance of mydriasis during cataract surgery. Ophthalmology 96: 585-588, 1989.

30. Bucci FA Jr and Waterbury LD: Aqueous prostaglandin E(2) of cataract patients at trough ketorolac and bromfenac levels after 2 days dosing. Adv Ther 26: 645-650, 2009.

31. Jin X, Jin H, Shi Y, Guo Y and Zhang H: Pyroptosis, a novel mechanism implicated in cataracts. Mol Med Rep 18: 2277-2285, 2018.

32. Davis BK, Wen H and Ting JP: The inflammasome NLRs in immunity, inflammation, and associated diseases. Annu Rev Immunol 29: 707-735, 2011.

This work is licensed under a Creative Commons Attribution-NonCommercial-NoDerivatives 4.0 International (CC BY-NC-ND 4.0) License. 